\title{
UM MODELO BASEADO EM AGENTES PARA TRANSFERÊNCIA DE TECNOLOGIA
}

\author{
Adriana Neves dos Reis \\ Universidade do Vale do Rio dos Sinos - UNISINOS \\ Av. Unisinos, 950 - São Leopoldo - RS \\ adriana.anreis@gmail.com \\ Guilherme Luís Roehe Vaccaro \\ Universidade do Vale do Rio dos Sinos - UNISINOS \\ Av. Unisinos, 950 - São Leopoldo - RS \\ guilhermev@unisinos.br
}

\begin{abstract}
Resumo
Transferência de Tecnologia (TT) é o negócio resultante da interação entre agentes que concordam em trocar posse, conhecimento e valor, beneficiando-se desta troca. De modo amplo, esses agentes estão organizados em quatro classes: empresa, centro tecnológico, universidade e governo. Em tal contexto, as negociações sofrem influência de fatores como: número de fornecedores de uma dada tecnologia, o conhecimento disponível em uma tecnologia específica, e a relação custo versus ganho esperado na operação. Assim, diferentes comportamentos emergem, competitivos e cooperativos, dependendo da relação desses fatores. Consequentemente, entender os mecanismos que inibem ou estimulam relações de TT pode oferecer suporte para a definição mais precisa de políticas setoriais e governamentais de estímulo a esse tipo de negociação. Uma abordagem para capturar e investigar esse tipo de fenômeno é a Modelagem e Simulação baseada em Agentes. Este artigo investiga a aplicação de um modelo baseado em agentes para extrair relações no contexto de TT, analisando ações para aumentar o número de negociações firmadas. O modelo considera dois tipos de agentes: empresa e universidade. Os resultados indicam a influência complexa do ambiente sobre a dinâmica das relações de TT.

Palavras-Chaves: Agentes; $\quad$ Simulação; Transferência de Tecnologia;
\end{abstract}

\begin{abstract}
Technology Transfer (TT) is the business resulting from the interaction between agents who agree to exchange ownership, knowledge and value, benefiting from this exchange. In generally, these agents are organized into four classes: firm, technology center, university and government. In this context, negotiations are influenced by factors such as number of a given technology suppliers, the knowledge available on a specific technology, and cost versus expected gain in the operation. Thus, different behaviors emerge, competitive and cooperative, depending on the relationship of these factors. Consequently, understanding the mechanisms that inhibit or stimulate TT relations can support a more precise definition of sectorial and government policies to stimulate this kind of negotiation. One approach to capture and investigate this kind of phenomenon is the agents-based modeling and simulation. This article investigates the use of an agent-based model based for extracting relations in the context of TT, analyzing actions to increase the number of signed negotiations. The model considers two types of agents: company and university. The results indicate the complex influence of the environment on the dynamics of relations TT.
\end{abstract}

Keywords: Agents; Simulation; Technology Transfer; 


\section{INTRODUÇÃO}

A Transferência de Tecnologia (TT), de universidades, centros de tecnologia ou empresas, na forma de conceitos vendáveis e com interesse de mercado tonou-se um tema de pesquisa importante [2]. Entre um conjunto de definições, [3] define TT como um evento que "ocorre onde pessoas fazem acordos a fim de obter melhores ferramentas, técnicas, materiais, etc. para realização de práticas" (p. 38). Esses pactos envolvem tipicamente diferentes classes de agentes: empresa, universidade, instituto tecnológico, e governo; os quais interagem em um ambiente complexo e dinâmico.

Essas interações exercem influência no ambiente, pois delas emerge um comportamento característico de negociação baseado nos atributos e objetivos de cada agente participante. Assim, compreender como a participação destes indivíduos contribui para a concretização de um negócio de TT trata-se de uma questão em aberto, uma vez que o conjunto de características individuais de cada agente associado a uma diversidade de fatores pode justificar seu comportamento em uma negociação de tecnologia [4] [5].

Uma estratégia para abstrair esse fenômeno é a Modelagem e Simulação baseada em Agentes, por ser uma técnica que opera no nível micro individual [6]. Um agente é a representação de um indivíduo com características específicas, o qual interage com outros agentes em um contexto compartilhado. Logo, o modelo descreve um sistema reativo que exibe certa autonomia para decidir quão bem deve executar uma tarefa a ele delegada [7]. Em [6] é apresentada uma revisão da adoção desta modelagem para o problema de difusão de tecnologia. Nela, os autores enfatizam a relevância dos modelos baseados em agentes como ferramenta para oferecer suporte à decisão baseada em dados empíricos.

De modo geral, existem diferentes modelos teóricos consolidados sobre mecanismos difusão da inovação [6] [10] [11]. Porém, o mesmo não ocorre na mesma diversidade quando o foco é específico em TT. Na literatura, poucos trabalhos abordam os mecanismos não lineares presentes no processo de TT. Do mesmo modo, pouco se tem explorado a influência de fatores específicos na negociação entre empresas e universidades desempenhando o papel de vendedor (fornecedor) e/ou comprador (receptor) de tecnologia. Igualmente, a função dos diferentes agentes no ecossistema de inovação não é claro, bem como sua influência em agregar valor social e público como resultado da TT [12].

Assim, este artigo investiga a aplicação da abordagem baseada em agentes para modelar e simular contextos de TT, de modo a identificar mecanismos que possam explicar esse processo bem como sustentar a definição de ações que estimulem a realização de negociações de TT. Para tanto, a seção 2 apresenta os aspectos relevantes do ambiente de TT, seguida da proposta de modelo baseado em agentes para TT, na seção 3. A seção 4 discute experimentos realizados para explorar a capacidade do modelo em simular mecanismos emergentes. Enfim, algumas considerações gerais sobre o estudo são apresentadas na última seção.

\section{O NEGÓCIO TRANSFERÊNCIA DE TECNOLOGIA}

TT é um mecanismo empregado pela firma para obter recursos tecnológicos necessários para criar inovação a partir de recursos externos, em uma relação de negócio envolvendo dois perfis: o fornecedor e o receptor [8]. Conforme [3], para entender como TT ocorre, pode-se adotar a metáfora do jogo, com peças e um tabuleiro, no qual a vitória é definida por um bom acordo, em que todas as peças envolvidas ganham. 
Para determinar políticas apropriadas para o desenvolvimento sustentado por práticas de inovação, os atores governamentais precisam entender como suas ações neste sentido podem influenciar a competitividade dos mercados internos, e, consequentemente, alavancar suas competências para competir em contextos mais amplos. No caso de TT, em especial como estratégia para inovação, identificar e compreender as relações entre os atores deste processo é um desafio para decidir por incentivos mais adequados.

Em uma economia global marcada por pressões de competição, a TT fornece meios para transformar tecnologias desenvolvidas em instituições públicas em produtos para comercialização [2]. Ainda, neste contexto, instituições acadêmicas e científicas possuem papel estratégico para dar suporte às firmas em suas necessidades para inovar [2][3]. Porém, o conhecimento a respeito do processo usado pelos empreendedores acadêmicos para acumular recursos e habilidades para obter sucesso no mercado é limitado [9].

O ecossistema da TT é concebido como um ecossistema de negócios, sendo este último entendido como uma comunidade econômica que "foca no cliente potencial e nas redes fornecedoras para empresas de alta tecnologia" [9]. Por exemplo, a função da universidade é criar conhecimento. Mas, recentemente, tem sido discutido o aumento das contribuições por parte do empreendedorismo das universidades [9]. Por outro lado, a observação indica que as empresas ora possuem recursos limitados ora carecem de sistemas estruturados de gestão para investir na compra e desenvolvimento de tecnologia. Em certos contextos, ainda soma-se a esses atores a compreensão de consumo e não de desenvolvimento da tecnologia. E, como em outros negócios, a expectativa ideal é a de realizar acordos com alto retorno sobre o investimento e riscos minimizados. Nesse amplo processo de negociação, acredita-se que a reputação do agente, seja universidade, centro de tecnologia ou empresa, pode estimular ou retrair aquelas interações que resultem em TT. E, do ponto de vista governamental, caracterizar a relação entre esses atores entende-se como um fator crítico para a tomada de decisão em políticas para estimular TT.

Desse modo, as interações no nível micro podem ser consideradas o ponto de partida para tais acordos, o que justifica o caráter promissor da abordagem baseada em agentes como abstração para TT. E modelar e simular o comportamento dos agentes de inovação, em diferentes cenários contextuais, pode contribuir de forma significativa para entender como ocorre a dinâmica de influência entre o nível micro e macro do ecossistema de inovação.

\section{MODELO DE TT BASEADO EM AGENTES}

Em um sistema adaptativo, "agentes representam as unidades básicas do processo da tomada de decisão" [13] (p. 55). Um modelo baseado em agentes utiliza uma abstração bottom up, baseada em agentes com funções simples. Além disso, quando estes indivíduos interagem, considerando suas funções, alguns comportamentos específicos emergem [7]. Dessa forma, neste contexto, é relevante associar simulação para investigar propriedades da dinâmica do processo estudado.

A abordagem baseada em agentes tem sido adotada em estudos para permitir a definição de modelos, os quais analisados em cenários reais, consequentemente, servem de base para a proposição de recomendações de ação nestes contextos [6]. No caso de modelos para TT, essas características são interessantes para a construção de um mapa das conexões lógicas em nível micro e macro [13]. Porém, na revisão da literatura, os elementos de abstração pouco são explorados em conjunto, desconsiderando os mecanismos de competição e cooperação associados ao ecossistema em que os atores interagem.

A Figura 1 apresenta os componentes-chave na constituição do modelo proposto. A 
base é o processo de TT, a partir do qual são selecionados elementos envolvidos nos caráter de competição e cooperação existente no agente e no ambiente, tendo como objetivo avaliar o impacto desses no número de negociações realizadas e que efetivamente resultaram em efetiva TT.

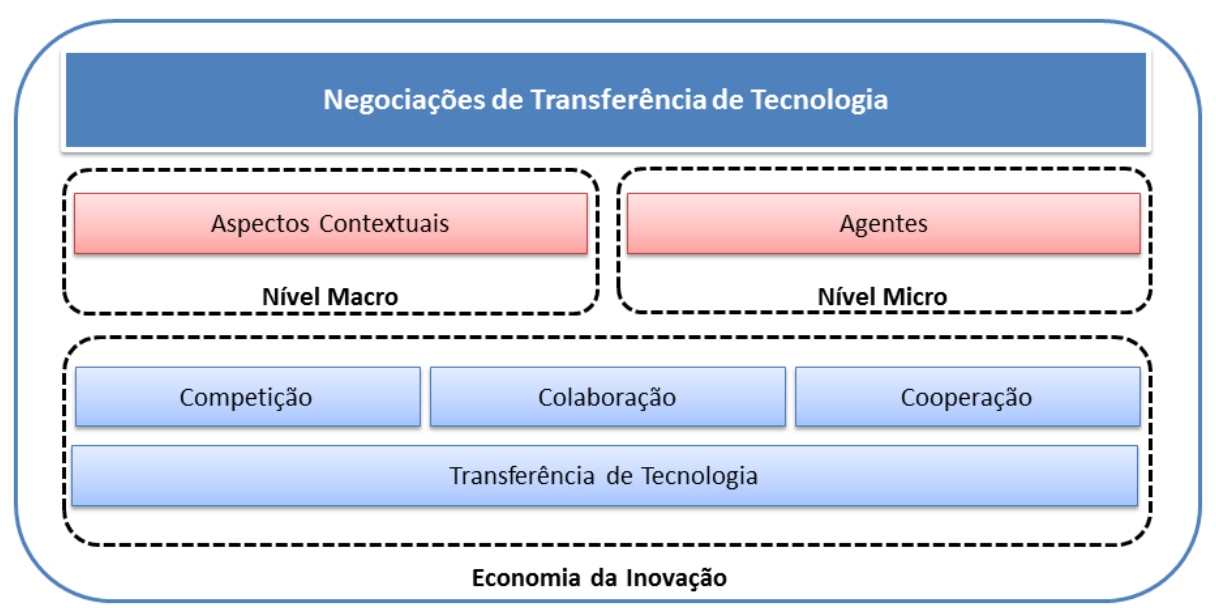

Figura 1 - Framework para Transferência de Tecnologia baseada em Agentes.

A Figura 2, por sua vez, trata-se de uma visão esquemática dos agentes participantes do processo de TT, suas características ligadas à tomada de decisão, e o contexto que as eles interagem. Neste ambiente, busca-se avaliar o grau de influência de dois aspectos: taxa de turbulência, que representa a intensidade de risco associada à operação de transferência devido ao elemento ambiental, e taxa de colaboração, que representa o quão diversificado é o grupo de agentes em sua intenção de fechar uma negociação.

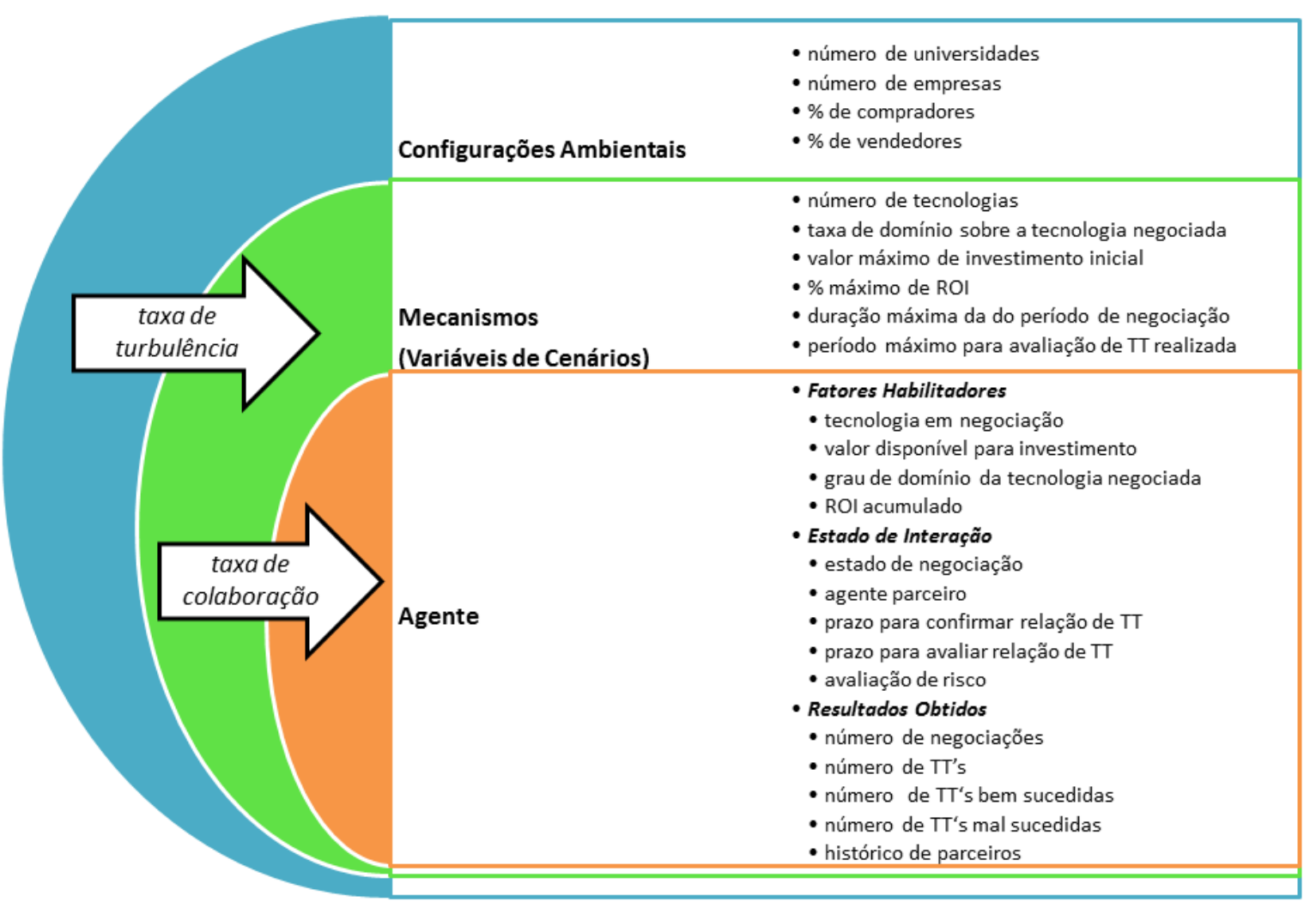

Figura 2 - Variáveis do modelo proposto organizados por nível de abstração. 
Os atores do processo de TT são, então, concebidos como agentes, segundo uma estrutura única, mas que desempenham diferentes papeis: universidade, centro de tecnologia ou empresa. Desse modo, todos os agentes possuem a mesma estrutura, a qual possui três classes de atributos: fatores habilitadores, estado de interação e resultados obtidos (Figura 2). A Tabela 1 apresenta as definições de cada um dos atributos, acompanhadas, se identificadas, de referências que o considerem como parte do ecossistema de TT. Consequentemente, a individualidade de cada agente é dada pelos valores de seus atributos, resultando em seu estado.

Tabela 1 - Definição dos atributos dos agentes de TT.

\begin{tabular}{|c|c|c|c|}
\hline & Atributo & Definição & Referências \\
\hline \multirow[t]{4}{*}{$\begin{array}{c}\text { Fatores } \\
\text { Habilitadores }\end{array}$} & $\begin{array}{l}\text { tecnologia em } \\
\text { negociação }\end{array}$ & $\begin{array}{l}\text { um código que representa uma } \\
\text { tecnologia hipotética }\end{array}$ & {$[14]$} \\
\hline & $\begin{array}{l}\text { valor disponível para } \\
\text { investimento }\end{array}$ & $\begin{array}{l}\text { a quantia disponível para investir } \\
\text { em TT }\end{array}$ & $\begin{array}{l}{[9],[11]} \\
{[15],[16]}\end{array}$ \\
\hline & $\begin{array}{l}\text { grau de domínio da } \\
\text { tecnologia negociada }\end{array}$ & $\begin{array}{l}\text { um valor aleatório que indica o } \\
\text { nível de conhecimento esperado } \\
\text { ou disponível sobre a tecnologia }\end{array}$ & $\begin{array}{l}{[9],[10],} \\
{[17],[18]}\end{array}$ \\
\hline & ROI acumulado & $\begin{array}{l}\text { o valor total obtido como retorno } \\
\text { do investimento em TT }\end{array}$ & {$[15],[17]$} \\
\hline \multirow{5}{*}{$\begin{array}{l}\text { Estado de } \\
\text { Interação }\end{array}$} & estado de negociação & $\begin{array}{l}\text { se o agente está interagindo em } \\
\text { um dado momento }\end{array}$ & {$[18]$} \\
\hline & agente parceiro & $\begin{array}{l}\text { o código que identifica o agente } \\
\text { com o qual está ocorrendo } \\
\text { interação }\end{array}$ & [19] \\
\hline & $\begin{array}{l}\text { prazo para confirmar } \\
\text { relação de TT }\end{array}$ & $\begin{array}{l}\text { um período pré-determinado para } \\
\text { decidir se a TT ocorrerá como } \\
\text { resultado da interação em } \\
\text { execução }\end{array}$ & [9] \\
\hline & $\begin{array}{l}\text { prazo para avaliar } \\
\text { relação de TT }\end{array}$ & $\begin{array}{l}\text { um período pré-determinado para } \\
\text { avaliar se os resultados obtidos } \\
\text { com a TT são satisfatórios e, } \\
\text { ainda, se a relação de contrato } \\
\text { deve ser mantida }\end{array}$ & [9], [18] \\
\hline & avaliação de risco & $\begin{array}{l}\text { um valor aleatório que indica a } \\
\text { taxa de risco associada à operação }\end{array}$ & - \\
\hline \multirow{5}{*}{$\begin{array}{l}\text { Resultados } \\
\text { Obtidos }\end{array}$} & número de negociações & número de interações realizadas & $\begin{array}{l}{[1],[16],} \\
{[17],[20]}\end{array}$ \\
\hline & número de TT's & número de acordos firmados & {$[1],[20]$} \\
\hline & $\begin{array}{l}\text { número de TT's bem } \\
\text { sucedidas }\end{array}$ & $\begin{array}{l}\text { número de TT's com resultado } \\
\text { positivo }\end{array}$ & {$[14]$} \\
\hline & $\begin{array}{l}\text { número de TT's mal } \\
\text { sucedidas }\end{array}$ & $\begin{array}{l}\text { número de TT's com resultado } \\
\text { negativo }\end{array}$ & {$[18]$} \\
\hline & histórico de parceiros & $\begin{array}{l}\text { conjunto dos agentes com quais o } \\
\text { agente já realizou TT }\end{array}$ & {$[18]$} \\
\hline
\end{tabular}


Os fatores habilitadores representam a condição do agente em participar e firmar acordos de Transferência de Tecnologia. O estado de interação descreve a condição do agente ao longo das interações dentro da comunidade de agentes. É importante destacar que no modelo proposto, o agente interage apenas com agente de tipo diferente do seu. Os resultados obtidos, por fim, denotam a experiência acumulada do agente ao longo da evolução da simulação. De forma geral, ainda é importante destacar que, na abordagem baseada em agentes, a maior ênfase é na simulação de cada interação social e não apenas o resultado de acordo [20].

A dinâmica de simulação do modelo é baseada na relação binária de TT que ocorre entre dois tipos de agentes distintos. Além disso, cada um desempenha um papel específico: comprador ou fornecedor de tecnologia [19], e a interação é motivada a partir de uma necessidade de obtenção de uma tecnologia específica. Nesta visão, a demanda de mercado é entendida como o fator direcionador da ocorrência de uma instância de TT. A função governamental, neste caso, não é considerada um agente, mas sua influência é representada por um conjunto de parâmetros relacionados ao ambiente em que ocorre a interação, além das taxas de turbulência e colaboração.

\section{EXPERIMENTOS}

Os experimentos apresentados nesta seção têm o objetivo de explorar adequação do modelo proposto para o contexto de TT, a fim de simular cenários emergentes na interação entres os atores universidade e empresa. Neste propósito, foram simulados 25 cenários variando taxa de turbulência e colaboração, ambas entre 0 (inexistente) e 1 (grau máximo).

A Figura 3 apresenta a visão geral do protótipo criado para a simulação do modelo implementado usando NetLogo 5.0, um ambiente de modelagem e programação multi-agente [21]. Os valores apontados na área de Configuração do Ambiente e Configuração dos Mecanismos de Interação, no ambiente de simulação da Figura 3, permanecem inalterados nos cenários desenvolvidos. A população de agentes possui 30 universidades, considerando os dados disponíveis no site da Coordenação de Aperfeiçoamento de Pessoal de Nível Superior (Capes) ${ }^{1}$ para o estado do Rio Grande do Sul. Em relação às empresas, são 500 agentes para abstrair um contexto próximo da indústria têxtil no estado do Rio Grande do Sul, de acordo com levantamento realizado pela Associação Comercial, Industrial e de Serviços de Novo Hamburgo, Campo Bom e Estância Velha (ACI ${ }^{2}$. Para fins da análise específica da relação entre a taxa de turbulência e colaboração, este artigo considera a variação apenas destes dois componentes do modelo para composição dos cenários de análise.

\footnotetext{
${ }^{1}$ Site: http://conteudoweb.capes.gov.br/conteudoweb/ProjetoRelacaoCursosServlet?acao=pesquisarRegiao

${ }^{2}$ Site: http://www.acinh.com.br/download/72
} 


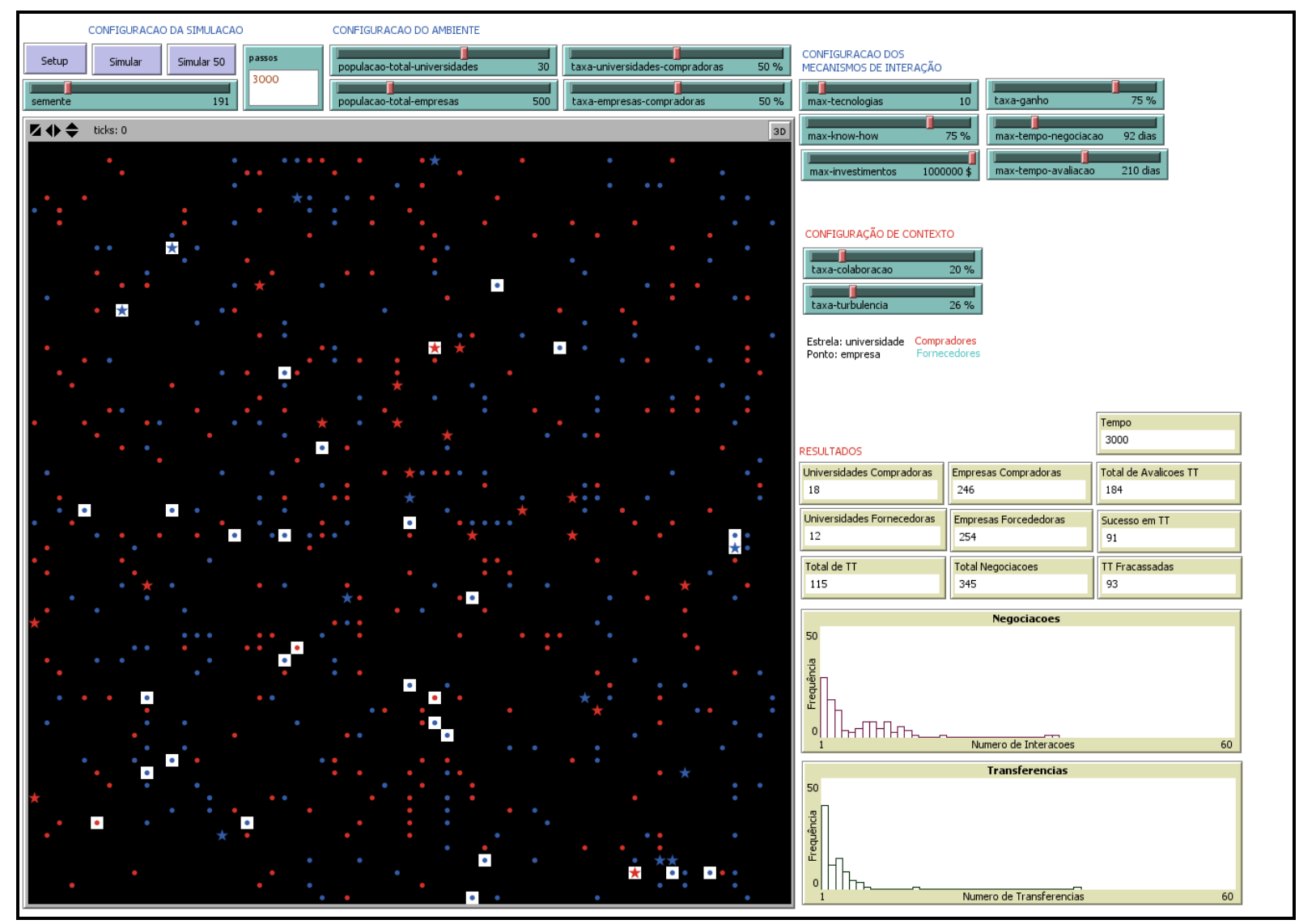

Figura 3 - Ambiente de simulação construído no NetLogo.

A Tabela 2 apresenta os resultados médios consolidados para 10 rodadas de cada um dos cenários, contemplando quatro métricas de desempenho para o contexto de TT: número de negociações de TT, número de TT efetivadas, número de avaliações positivas das parcerias de TT e número de avaliações negativas das parcerias de TT. Uma avaliação negativa não é suficiente para o rompimento de uma parceria, mas contribui para que essa ocorra ao longo do tempo. Além disso, cada parceria de TT passa por avaliação diversas vezes conforme o parâmetro período máximo para avaliação de TT realizada.

Conforme os dados da Tabela 2, observa-se que o número de avaliações, sejam positivas ou negativas, cresce conforme aumenta a taxa de colaboração. Tal aspecto evidência que, para fins do modelo proposto, a obtenção de sucesso ou fracasso em uma avaliação é independente do grau de oportunidade para TT, tanto em relação à conjunta do ambiente quanto ao perfil dos agentes.

Em relação ao número de transferências de tecnologia e ao número de negociações, os gráficos da Figura 4 apresentam as curvas de comportamento do impacto da taxa de colaboração para diferentes contextos de turbulência do ambiente. Conforme pode ser observado na imagem (a), o aumento da taxa de colaboração faz reduzir o número de negociações, o que pode ser explicado pelo fato desse aumento proporcionar o fechamento do acordo de TT mais rápido, reduzindo o número de tentativas de negociação. Além disso, percebe que no caso de turbulência inexistente, a curva se distancia das demais. Com o objetivo de visualizar em maior detalhe as curvas que se aproximam, o gráfico (b) exclui a linha referente à turbulência igual a zero, e altera a escala do eixo do número de negociações. Conforme o mesmo, é possível identificar que há entrelaçamento entre as mesmas para os 
diferentes valores de turbulência, o que evidência a possibilidade de fenômenos emergentes baseados na configuração dos agentes, e não apenas relacionados diretamente às taxas de colaboração e turbulência.

Tabela 2 - Resultados da Simulação para comparação turbulência X colaboração.

\begin{tabular}{|l|l|l|c|c|c|}
\hline \multirow{2}{*}{$\begin{array}{c}\text { Turbulência } \\
\text { (percentual) }\end{array}$} & \multirow{2}{*}{$\begin{array}{c}\text { Colaboração } \\
\text { (percentual) }\end{array}$} & $\begin{array}{c}\text { Total de } \\
\text { Negociações }\end{array}$ & $\begin{array}{c}\text { Total de } \\
\text { Transferências } \\
\text { de Tecnologia }\end{array}$ & $\begin{array}{c}\text { Total de } \\
\text { Avaliações } \\
\text { com Sucesso }\end{array}$ & $\begin{array}{c}\text { Total de } \\
\text { Avaliações } \\
\text { com Fracasso }\end{array}$ \\
\hline 0 & 0 & 1115,5 & 0 & 0 & 0 \\
\hline 0 & 0,25 & 793,5 & 163 & 132 & 141 \\
\hline 0 & 0,5 & 662,5 & 284,5 & 219,5 & 231,5 \\
\hline 0 & 0,75 & 607,5 & 385 & 289 & 301 \\
\hline 0 & 1 & 522,5 & 447,5 & 303 & 324 \\
\hline 0,25 & 0 & 640,5 & 0 & 0 & 0 \\
\hline 0,25 & 0,25 & 484,5 & 102,5 & 92 & 88,5 \\
\hline 0,25 & 0,5 & 408 & 168,5 & 126 & 136,5 \\
\hline 0,25 & 0,75 & 347 & 221,5 & 176,5 & 177,5 \\
\hline 0,25 & 1 & 317,5 & 261 & 206 & 201 \\
\hline 0,5 & 0 & 602 & 0 & 0 & 0 \\
\hline 0,5 & 0,25 & 461,5 & 92 & 72,5 & 80,5 \\
\hline 0,5 & 0,5 & 399,5 & 158 & 136 & 128 \\
\hline 0,5 & 0,75 & 338 & 216,5 & 168 & 172,5 \\
\hline 0,5 & 1 & 293,5 & 254 & 199 & 194 \\
\hline 0,75 & 0 & 666 & 0 & 0 & 0 \\
\hline 0,75 & 0,25 & 477 & 97 & 99 & 82 \\
\hline 0,75 & 0,5 & 426,5 & 176 & 140,5 & 139 \\
\hline 0,75 & 0,75 & 349,5 & 222 & 165,5 & 173,5 \\
\hline 0,75 & 1 & 322,5 & 281 & 205 & 206,5 \\
\hline 1 & 0 & 639,5 & 0 & 0 & 0 \\
\hline 1 & 0,25 & 504,5 & 102 & 86 & 89 \\
\hline 1 & 0,5 & 421 & 171,5 & 126 & 139,5 \\
\hline 1 & 0,75 & 358 & 229 & 169 & 178,5 \\
\hline 1 & 1 & 328 & 287 & 198,5 & 211 \\
\hline & & & & & \\
\hline & & & & & 0 \\
\hline
\end{tabular}

Ainda na Figura 4, os gráficos (c) e (d) apresentam o efeito da turbulência e da colaboração no número de TT realizado. Semelhante ao comportamento no número de negociações, no cenário de turbulência inexistente a curva se distancia consideravelmente das demais em (c). Ainda, quando excluída essa curva da visualização, em (d), também é possível verificar que não há uma separação evidente das curvas dos diferentes cenários para turbulência de até 0,75 . No caso de grau máximo de colaboração, porém, maiores turbulências implicaram maior número de TT. 


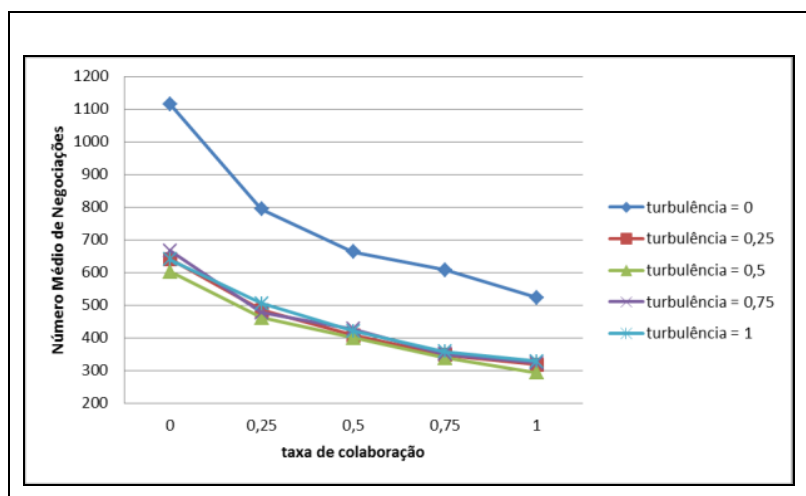

(a)

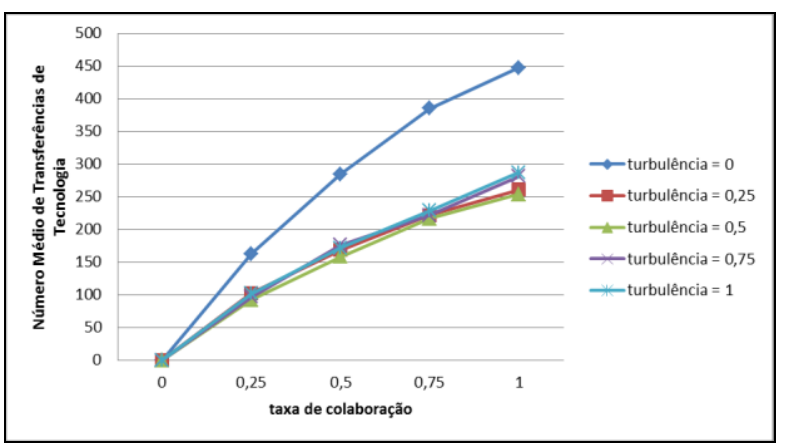

(c)

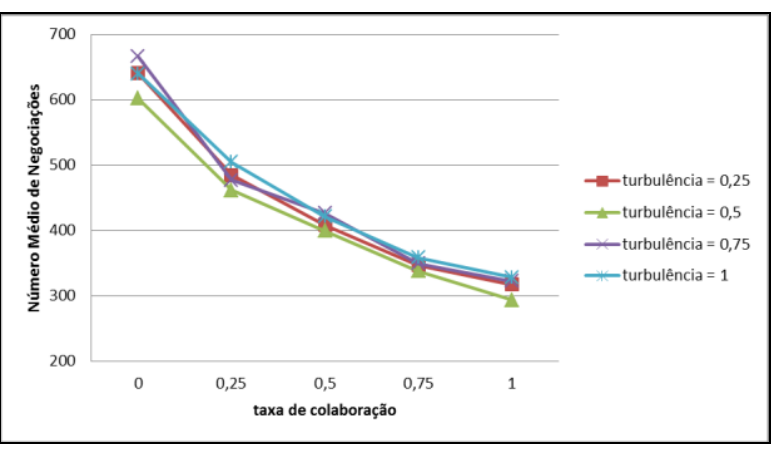

(b)

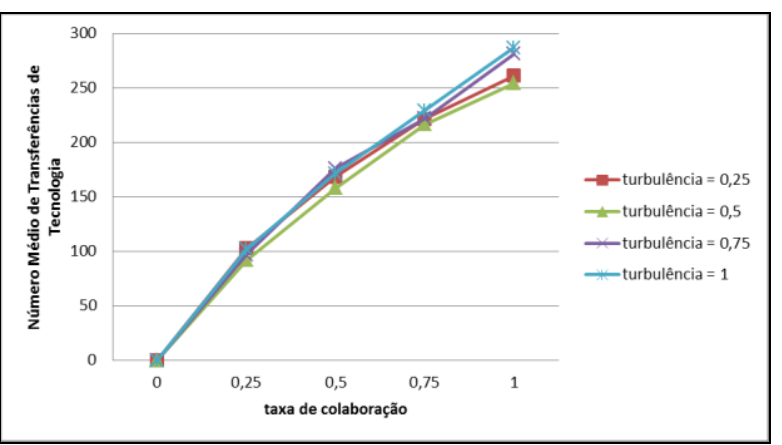

(d)

Figura 4 - Resultados de Simulação para Cenários de turbulência X colaboração.

Para caracterizar o favorecimento do ambiente para a ocorrência de TT, o gráfico da Figura 5 apresenta, para cada cenário, a taxa média de número de TT efetivadas por número de negociações realizadas. Conforme se pode constatar, do ponto de vista macro, parece haver uma relação direta entre colaboração e turbulência, trazendo indícios de que é relevante uma análise detalhada da dimensão dos impactos individuais e em conjunto de ambas. A partir dela espera-se identificar se a colaboração pode ser um fator mais impactante no número de TT em diferentes cenários de conjuntura do contexto.

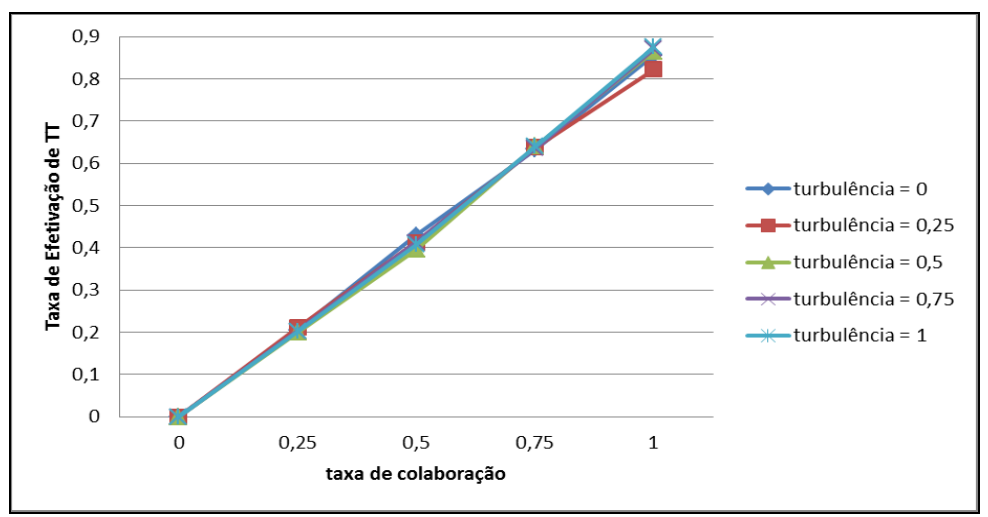

Figura 5 - Resultados de Efetivação de TT em Cenários de turbulência X colaboração. 
As simulações apresentadas, ainda que simples, permitem evidenciar os efeitos do cenário complexo do ambiente de TT sobre o número de negociações realizadas. A compreensão do efeito dinâmico das variáveis ambientais e contextuais sobre a TT, junto ao comportamento dos agentes em reação ao meio, permite estabelecer proposições e discussões aprofundadas sobre o tema, bem como testar hipóteses de forma facilitada em relação a modelos estáticos. A exploração do modelo proposto como suporte à tomada de decisão requer, portanto, refinamentos e inputs oriundos de estudos empíricos sobre o contexto de TT e seus atores.

\section{CONSIDERAÇÕES FINAIS}

Compreender o comportamento dos atores de uma relação de TT e seus critérios para tomada de decisão é um desafio na perspectiva tanto de estudos que abordam inovação quanto de estudos de simulação. O modelo de TT proposto evidencia que Modelagem e Simulação baseada em Agentes possuem características potenciais como ferramenta de investigação do ambiente de TT e, consequentemente, oferece suporte para tomada de decisão que estimule este tipo de prática de inovação.

O modelo apresentado, em fase preliminar, apresenta algumas características representativas dos agentes e seus padrões comportamentais em nível micro. Outras características ainda necessitam ser implementadas, de modo a incrementar a aderência dos agentes ao comportamento dos atores reais. Além disso, o próprio modelo pode ser ampliado para considerar características específicas, tais como: políticas e comportamentos de mercado, políticas de inovação, influência geográfica, ou outros fatores comparativos e competitivos.

Assim, o estudo está direcionado a investigar, nas próximas etapas, a adequação dos elementos estruturais da teoria de agentes às abstrações dos agentes de Transferência de Tecnologia, bem como os mecanismos de interação nestes contextos. Para, dessa forma, identificar a capacidade do modelo de agentes em representar a dinâmica de decisão em TT.

\section{REFERÊNCIAS BIBLIOGRÁFICAS}

[1] A. Heshmati and F. Lenz-Cesar, "Policy Simulation of Firms Cooperation in Innovation," no. 357, 2014.

[2] D. B. Audretsch, E. E. Lehmann, and M. Wright, "Technology transfer in a global economy," J. Technol. Transf., vol. 39, no. 3, pp. 301-312, 2014.

[3] P. L. Speser, The Art \& Science of Transfer Technology. Hoboken: John Wiley \& Sons, 2006.

[4] X. Liu and S. White, "Comparing innovation systems : a framework and application to China 's transitional context \&,," Res. Policy, vol. 30, pp. 1091-1114, 2001.

[5] Impactos tecnológicos das parcerias da Petrobras com universidades, centros de pesquisa e firmas brasileiras. 2013.

[6] E. Kiesling, M. Günther, C. Stummer, and L. M. Wakolbinger, "Agent-based simulation of innovation diffusion: A review," Cent. Eur. J. Oper. Res., vol. 20, no. 2 , pp. 183-230, 2012. 
[7] R. H. Bordini, J. F. Hübner, and M. Wooldridge, Programming multi-agent systems in AgentSpeak using Jason, vol. 8. John Wiley \& Sons, 2007.

[8] J. Gonzalez Sabater, "Manual de trasferencia de tecnología y conocimiento," pp. 1$124,2010$.

[9] M. Wright, "Academic entrepreneurship, technology transfer and society: where next?," J. Technol. Transf., pp. 1-13, 2012.

[10] P. Morone and R. Taylor, "Proximity, knowledge integration and innovation: An agenda for agent-based studies," J. Evol. Econ., vol. 22, no. 1, pp. 19-47, 2012.

[11] R. Leite and A. a C. Teixeira, "Innovation diffusion with heterogeneous networked agents: A computational model," J. Econ. Interact. Coord., vol. 7, no. 2, pp. 125-144, 2012.

[12] B. Bozeman, H. Rimes, and J. Youtie, "The evolving state-of-the-art in technology transfer research: Revisiting the contingent effectiveness model," Res. Policy, vol. 44, no. 1, pp. 34-49, 2014.

[13] N. Nan, R. Zmud, and E. Yetgin, "A complex adaptive systems perspective of innovation diffusion: an integrated theory and validated virtual laboratory," Comput. Math. Organ. Theory, vol. 20, no. 1, pp. 52-88, 2013.

[14] M. Blom, F. Castellacci, and A. Fevolden, "Defence firms facing liberalization: innovation and export in an agent-based model of the defence industry," Comput. Math. Organ. Theory, no. 123, 2014.

[15] a. S. Mohamed, S. M. Sapuan, M. M. H. Megat Ahmad, a. M. S. Hamouda, and B. T. Hang Tuah Bin Baharudin, "Modeling the technology transfer process in the petroleum industry: Evidence from Libya," Math. Comput. Model., vol. 55, no. 3-4, pp. 451-470, 2012.

[16] S. Lee, W. Kim, Y. M. Kim, and K. J. Oh, "Using AHP to determine intangible priority factors for technology transfer adoption," Expert Syst. Appl., vol. 39, no. 7, pp. 63886395, 2012.

[17] G. Petroni, C. Verbano, B. Bigliardi, and F. Galati, "Strategies and determinants for successful space technology transfer," Space Policy, vol. 29, no. 4, pp. 251-257, 2013.

[18] P. D’Este, E. Castro-Martínez, and J. Molas-Gallart, "Documento de base para un ' Manual de Indicadores de Vinculación de la universidad con el entorno socioeconómico ': un marco para la discusión," p. 42, 2009.

[19] N. Khabiri, S. Rast, and A. A. Senin, "Identifying Main Influential Elements in Technology Transfer Process: A Conceptual Model," Procedia - Soc. Behav. Sci., vol. 40, pp. 417-423, 2012.

[20] C. Watts and N. Gilbert, Simulating Innovation: Computer-based Tools for Rethinking Innovation. Edward Elgar Publishing, 2014.

[21] A. B. Shiflet and G. W. Shiflet, "An introduction to agent-based modeling for undergraduates," Procedia Comput. Sci., vol. 29, pp. 1392-1402, 2014. 